International Journal of Education (IJE), Vol. 3, No. 3, September 2015

\title{
Organising SUbJECT MATERIAL IN ACCORDANCE WITH UBIQUITOUS STUDENT STATUS
}

\author{
Anandi Giridharan, Pallapa Venkataram* \\ Department of Electrical Communication Engineering, \\ Indian Institute of Science, Bangalore-12, India
}

\begin{abstract}
Ubiquitous learning allows students to learn at any time and any place. This educational activity is possible to be performed by various types of students and to operate on various devices, networks and environments, where the system understands the study pattern and behaviour of the students. Adaptivity plays an important role in Ubiquitous learning, aiming at providing students with adaptive and personalized learning material and information at the right place and the right time. Student's history logs is automatically created and maintained by the student history database that maintains student's history of subject content requested. This offers information on student's hardware capabilities, students preferences, knowledge level and student status. This information can be utilized to respond to new student's request with subject content created from previous similar request. A Ubiquitous learning student model aims to identify students needs, characteristics and situations. We use C-IOB (Context-Information, Observation and Belief) model to process the context of the student, formulate the observation and use the observations to generate beliefs. The belief generated by $C-I O B$ model is based on adaptation decision and subject analysis from student history database, which are able to detect the real-world learning status of students. Designed method has been illustrated for students with divergent knowledge levels, by considering complete course material of a subject, Communication Protocols offered at graduate level.
\end{abstract}

\section{KEYWORDS}

Ubiquitous Learning, Ubiquitous student model, C-IOB Model, history logs.

\section{INTRODUCTION}

Ubiquitous learning extends e-learning by leading the notion of anytime and anywhere to reality, aiming at providing students with opportunities to learn and take in educational concepts in their living environments. In Ubiquitous learning, education is happening all around the student but the student may not even be aware of the learning process. Students do not have to do anything in order to learn but just need to activate their registered device(s). Ubiquitous learning environment considers student's current location along with environmental context (Chang and Chang, 2006; Ogata and Yano, 2004). Problem based learning is on approach based on learner-centered and targets on putting students in real-world problem situations that can enhance the students motivation (Haith-Cooper, 2000). In addition, problem based learning also promotes the skills and knowledge required in problem solving by working collaboratively/cooperatively in small groups (Duch et al., 2001; Savery and Duffy, 1995). Students may also feel more connected with each other as voice and images can support the students social presence and help students feel more of a community of learners (Garrison et al., 2000) using multimedia input. The social network service aims at integrating social network features into the ubiquitous learning environment (Grunwald Associates LLC, 2007). Teachers get a better understanding about student's progress, knowledge level and other characteristics from student model. The designed

DOI : 10.5121/ije.2015.3301 
student model includes information about the student, based on the system's beliefs about him/her for the period of context. In order to provide students with learning material, the Ubiquitous learning environment is clubbed with the learning management system, Moodle (2008) one of the most appropriate environments for adaptivity (Graf List, 2005). Moodle helps in adapting most appropriate subject content to the student by considering the student's learning styles M. Wisl (1993). Main features of the Ubiquitous learning environment are that it considers the student's current location, (Chen et al (2008)). The rest of the paper is organized as follows, Section 2. Discusses the structure of a Ubiquitous Learning System and its main components; Section 3. Gives details on Ubiquitous Student Model; Section 4. describes student status analysis by C-IOB model, Section 5 discusses casual subject domain modelling. Section 6. discusses regarding tracking students' subject content based on historical web mining, Section 7 discusses evaluation of Ubiquitous Learning system and Section 8 gives conclusions.

\section{UBIQUITOUS-LEARNING SYSTEM}

In this section, we discuss functioning of a Ubiquitous Learning system by describing its components. The overview of the Ubiquitous Learning System is shown in the (Figure 1). The Ubiquitous Learning system is context aware; that is system senses the physical context of the student and appropriately provides learning support based their real world status. Student Model evaluates student' learning status and performance on a particular subject. Based on the outcome of the Student Model, Ubiquitous Learning system determines subject material, in accordance with Ubiquitous student status.

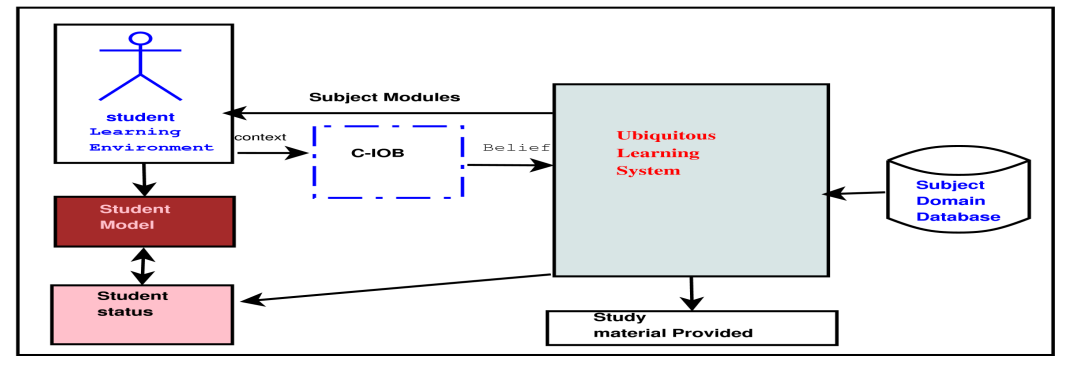

Figure 1 AN OVERVIEW OF A UBIQUITOUS LEARNING SYSTEM

\section{UbiQuitous-STUdent Model}

The Ubiquitous Student Model plays a important role, storing and updating the appropriate information about students which is needed in order to provide adaptivity. The student model includes all information about students like student profile, access to course content, progress in performance, interests and knowledge level, learning styles, problem-solving abilities, social closeness, and location etc.

Figure 2. shows the architecture of Ubiquitous Student Model using (C-IOB) ContextInformation, Observation, Belief model. We have used the C-IOB model to examine the context data from physical, system, application, and social domains. The beliefs developed about the students are primitive in most theories of decision making so that applications can use these beliefs in addition to student's history logs for providing intelligent adaptive learning service. We discuss the components of Ubiquitous Student Model. 


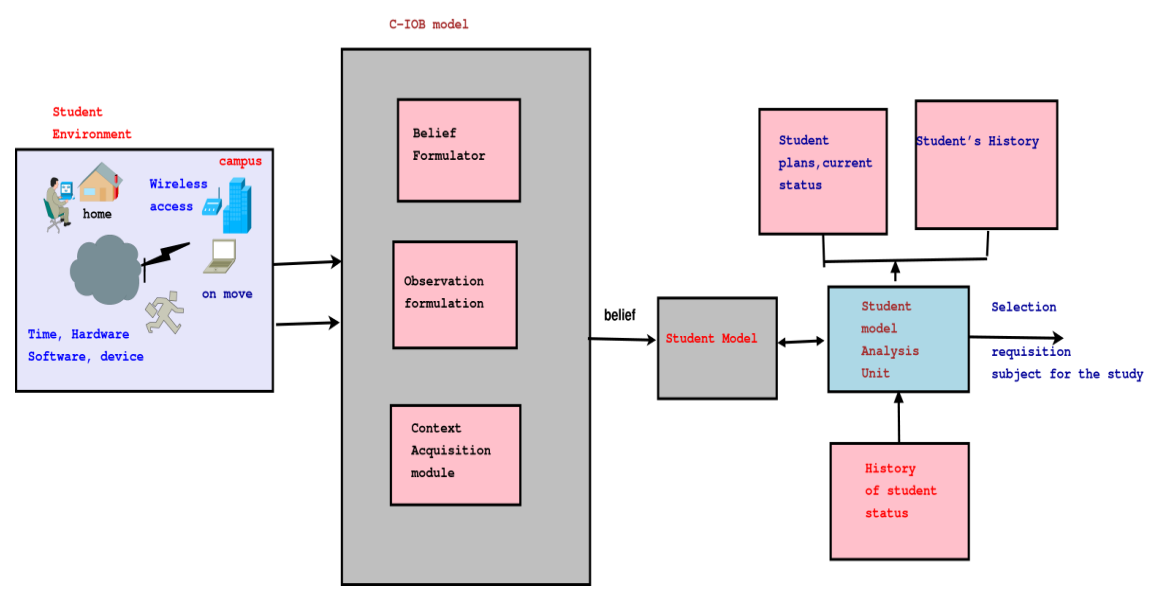

Figure 2. C-IOB based Ubiquitous Student Model

\subsection{Context information:}

Contest Information is variation in the context value, when the student is executing a particular study process. Clearly-defined context information is explaining the context space of an entity with respect to an environment with specific values. The context acquisition module acquires the context information from four surrounding environments.

Physical environment context in learning environment: if the student is moving towards a room to join the group discussion through video conference, the nearest functional objects such as a CCD camera and display equipment are located by the system immediately. In case if the student moves to another room, the system will change hardware devices accordingly based on students context, so that the video conference can be seamlessly continued (M. Takemoto,et al (2004).

System environment context: For the student, who is learning to identify the types of plants on campus, relevant information concerning the features of each type of plant is provided automatically based on his/her location and the plants around him or her.

Application context: Outdoor GPS receivers or indoor positioning system provides location information for mobile devices Active Badge devices, floor-embedded presence sensors may help in tracking the location of student or detecting their presence.

Social context: Related Subject contents for student in collaborative learning based on their location, goal preference and competency.

\subsection{Observation formulator:}

The observation formulator module creates methodically the observations based on the present context details of the student. An observation is made by discovering individual context states and how the student behaves in these states. For example, the context parameters: Time, hardware device, location, application, activity could produce observations like identifying student's preference, hardware device used, multimedia choice used that helps in browsing for specific course content, chatting with specific groups of students etc. 


\subsection{Belief formulator:}

The belief formulator is a component of C-IOB which gathers various temporal and spatial context information parameters from the environment (Pushpa and Venkataram, Pallapa (2011)). Formulating beliefs using context detail is one of the important features of the mind in knowledge representation (Sejong Gu, Hongzhou Shi, Jian Ye, Zhenmin ZhuM. Takemoto, et al (2007)). In order to provide personalization services, Ubiquitous Learning System should be able to make some inferences about their student, and this can be done by making some assumptions (beliefs based on Observation) about students based on their interaction with the system. The belief generated by C-IOB model is based on student preference, hardware device, knowledge level, interest etc, that helps to discover the appropriate subject content based on real time status. The belief formulation is given in (Algorithm 1).

\section{Algorithm 1 Pseudo Code for Belief}

\section{1: Begin}

2: Input : Set of context information of Students

3: Output : Belief $b_{i}$

4: Initialize the Students Context Information environment

5: Get the Students Context Information(CI).

6: Let $\mathrm{CI}_{(\mathrm{n})}=\left\{\mathrm{ci}_{1}, \mathrm{ci}_{2}, \ldots, \mathrm{ci}_{\mathrm{k}}\right\}$ is the set of current context information parameter values collected at particular instant of time.

7: Generate the context information parameter set $\mathrm{CI}_{(\mathrm{n})}$ using CI.

8: Generate the observations set $\mathrm{OB}$ using $\mathrm{CI}_{(\mathrm{n})}$

9: $\mathrm{B}$ is the set of beliefs generated from the list of $\mathrm{OB}$.

10: Let $b_{i}$ is the selected belief from set $B$.

11:Return bi .

\section{2:END}

\section{Student Status AnAlysis By C-IOB MODEL}

In our work, we consider above context information by using several devices like sensors, RFID, camera etc. The acquired context information will be formed into observation. An observation is formed by identifying individual context states and how the student behaves in these states. For example, the context parameters: time, device, location, activity could produce observations like browsing for study information, checking for lectures, demos, listening to lectures, talks etc. The Observations formulated on various behaviours of the students will be deduced into beliefs.

\begin{tabular}{|c|c|c|}
\hline Context Information & Observation & Belief \\
\hline $\begin{array}{l}\text { Activity -Movernent of student a } \\
\text { that instant time t1 } \\
\text { Walking, climbing stairs, sitting }\end{array}$ & Student is moving tow ards class room & $\begin{array}{l}\text { Sudent is moving to cl assroom to attend } \\
\text { lecture. }\end{array}$ \\
\hline $\begin{array}{l}\text { Student is at location }(x 1, y 1) \text { at } \\
\text { time } 11\end{array}$ & $\begin{array}{l}\text { At time instart } t 1 \text { student is in that } \\
\text { location }\end{array}$ & $\begin{array}{l}\text { Student is spending more time in class room } \\
\text { in discussion }\end{array}$ \\
\hline Student device-PDA, cell/phone & $\begin{array}{l}\text { Sensor } O N \text { and student movement } \\
\text { is very slow }\end{array}$ & $\begin{array}{l}\text { Student is using PDA and accessing cour se } \\
\text { contents. }\end{array}$ \\
\hline
\end{tabular}

FIGURE 3. STUDENT'S STATUS ANALYSIS USING C-IOB MODEL 
The belief formulator collects various temporal and spatial context information parameters according to time and place and also timely execution of activities as seen in Figure 3. Ubiquitous Learning system offers instant adaptive support to students by analysing the needs of the individual students from different perspectives like knowledge levels, profiles, performance etc.

\section{Casual SubJect domain Modelling:}

Generally given topic or course may be divided into set of Modules. Let $\mathrm{S}$ be the course which is divided into $n$ modules, $S=\left\{M_{1}, M_{2}, M_{3} \ldots M_{n}\right\}$. These modules has been interlinked, based on the subject material placements in each of the module. The modules are arranged in the order of degree of difficulty and concepts had five question $\mathrm{Q}_{1}, \mathrm{Q}_{2} \ldots \mathrm{Q}_{5}$ each. Depending on belief rules to student model, subject material is organized. Figure 4. shows representation of subject domain tree to represent various student's knowledge level. It generates study sequences matching the knowledge level and its student model.

EXAMPLE 1.Communication Protocol course subject was designed with concepts having different weight based on student knowledge level. The cognitive contribution of the subject is obtained by the following relation: The modules are arranged in the order of degree of difficulty. DD (M1 ) < DD (M2 )...... < DD (Mn ), where DD is degree of difficulty of the module. The weights of the module are given by $\mathbf{M}_{\mathrm{wi}}=\mathbf{x} * \mathbf{i}, \mathbf{i} \in \mathbf{1} \ldots . . . \mathbf{n}-\mathbf{1}$ where value $\mathrm{x}$ depends on the level of difficulty of the modules, that may vary from 0 to 30 for Novice level, 31 to 60 for average level, 61 to 100 for expert level and so on. The interlinked weight increases as the modules gets more advanced. A node in course content indicates a study strategy the student is suppose to follow.

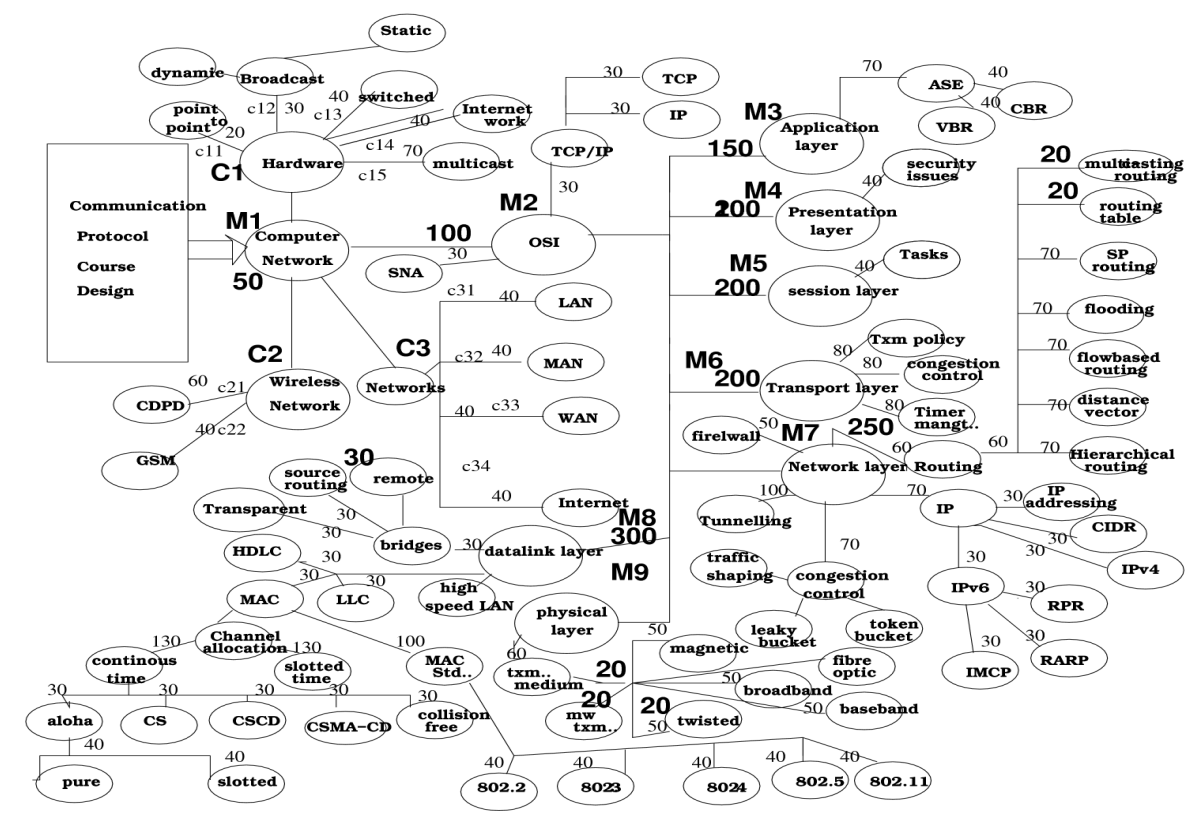

Figure 4 Student's study pattern on Communication Protocol course 


\subsection{Variables to measure the student's knowledge}

To measure the student' knowledge, subject expert use variables at different levels of granularity that vary between 0 to 1 (Figure 5) shows granularity hierarchy for subject communication protocols and appropriate student knowledge level required to access it.

\begin{tabular}{|l|l|c|c|}
\hline Subject & $\begin{array}{l}\text { Module/Concept } \\
\text { Weight }\end{array}$ & $\begin{array}{l}\text { Knowledge } \\
\text { level }\end{array}$ & $\begin{array}{c}\text { Student Category } \\
\text { eligble to access }\end{array}$ \\
\hline $\begin{array}{l}\text { Communication } \\
\text { Protocols }\end{array}$ & $0-30$ & $0-0.3$ & Novice student \\
& $31-60$ & $0.31-0.60$ & Average Student \\
& $61-100$ & $0.61-1.0$ & Expert Student \\
\hline
\end{tabular}

FIGURE 5 GRANULARITY HIERARCHY FOR SUBJECT COMMUNICATION PROTOCOLS

\section{TRACKING STUDENT'S SUBJECT CONTENT BASED ON HISTORICAL WEB MINING}

Student's interests may be described by the student's behavior for the past context. Our system learns about present student interests that includes recent relevant observations. System searches for the past history logs where the student has demonstrated a similar set of interests and try to learn a more precise description of the current student'/s interest. Student's behavior, seen as set of implicit feedback indicators such as past click history, click through data, and browsing features. Clustering algorithm, which has been effectively applied in web usage mining and personalized service applications (P.Boopathi, Prof.M.Sadish Sendil, Dr. S.Karthik (2010) ).

\subsection{Preprocessing the history Log to extract student's sessions}

The history access log of students is a very good reference of all subject contents (Subject contents URLs) accessed by students. Each log entry consists of the following Information: student's course access time, course IP address, course URL viewed etc. Algorithm 2. was used on a set of course sessions pre-processed from the history log data.

\section{Algorithm 2. Clustering Algorithm in Ubiquitous Student sessions}

\section{1: Begin}

2:Input: $\mathrm{x}_{\mathrm{j}}$, Student's sessions, $\mathrm{N}_{\mathrm{mi}} \mathrm{n}$ : Clusters-3

(Novice, Average, Expert), $\mathbf{W}_{\mathrm{ti}}$ : subject weightage scale (0-100).

3: Output $\mathbf{p}_{\mathbf{i}}$ : Student's profiles (sets of $\log$ URLs).

4: Encode binary session vectors $\mathrm{x}_{\mathrm{j}}$

5: Set current Knowledge Level KL=Novice Level

6: Apply clustering to entire data set.

7: Novice: $\mathrm{N}_{\mathrm{i}}<\mathrm{N}_{\min }$ or subject weightage scale $0 \leq \mathbf{W}_{\mathrm{ti}} \leq 30$.

8: Increment Knowledge Level: $\mathrm{KL}=$ Average Level

9: Apply clustering to entire data set.

10: Average: $\mathrm{N}_{\mathrm{i}}<\mathrm{N}_{\text {min }}$ or subject weightage scale $31 \leq \mathbf{W}_{\mathbf{t i}} \leq 60$

11: Increment Knowledge Level: KL=Expert Level

12: Apply clustering to entire data set.

13: Expert: $\mathrm{N}_{\mathrm{i}}<\mathrm{N}_{\min }$ or subject weightage scale $61 \leq \mathbf{W}_{\mathbf{t i}}<100$.

14: END 


\subsection{Summarizing Sessions clusters into student's profiles}

In clustering, grouping of sequences of page requested by students called session, which is the set of pages that are visited by a student within a predefined period of time. Algorithm is used to cluster students with same profiles and interest and knowledge level. Adaptive subject content is provided systematically to each category of students in ubiquitous learning. After automatically grouping session into different clusters, the session categories in terms of student profile vectors. $\mathrm{p}_{\mathrm{i}}$ : the $\mathrm{k}^{\text {th }}$ component, weight of this vector $\left(\mathrm{p}_{\mathrm{ik}}\right)$ captures the relevance of $\mathrm{url}_{\mathrm{k}}$ in the $\mathrm{i}^{\text {th }}$ profile as estimated by the conditional probability that $\operatorname{url}_{k}$ is accessed in the sessions belonging to $i^{\text {th }}$ cluster. The model is further extended to a robust profile based on robust weights that assign only sessions with high robust weight a cluster's core. These profiles give a cleaner description of the student interests as shown in (Figure 6).

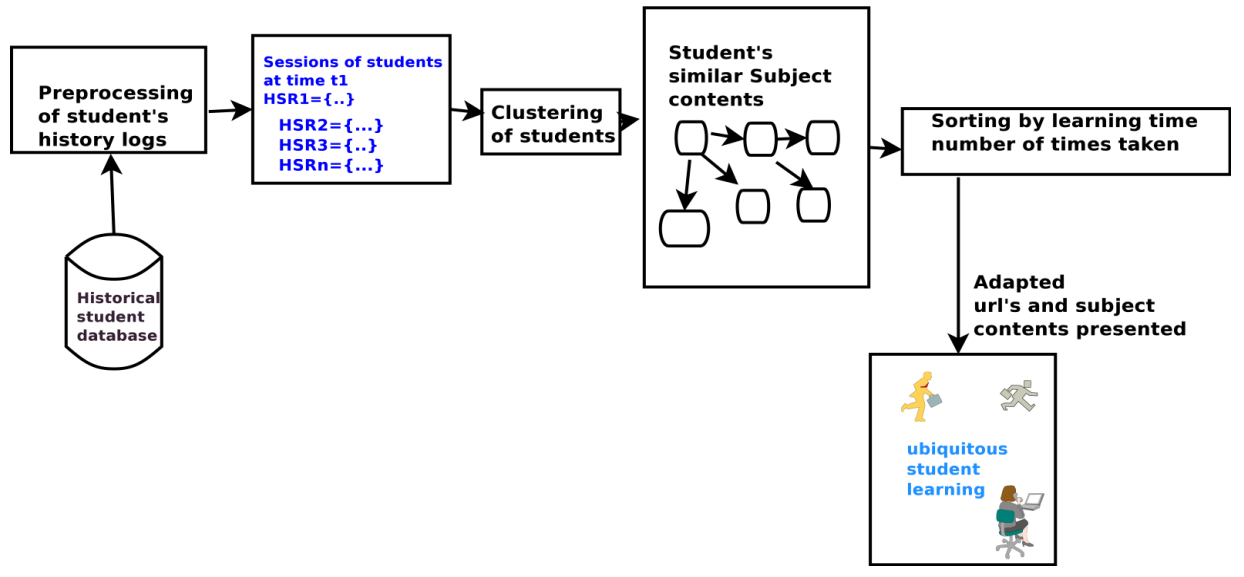

Figure 6 Adaptive subject content recommendation

\section{EVALUATION OF UBIQUITOUS LEARNING SYSTEM}

To assess the performance of our proposed Ubiquitous Learning System, we used simulated students on 15 modules for six months and studied student's performance in different locations both indoor and outdoor environment. Performance was evaluated by the experiments in terms of the personalized learning content delivering process, including the subject content adaptation process, both in indoor house, classroom and outdoor environment like garden, campus, travelling. ( Eva Millán, *, Luis Descalço , Gladys Castillo , Paula Oliveira , Sandra Diogo , 2013). Simulation was conducted in blocks of varying knowledge levels, including a variety of different contexts. An initial clustering based on different knowledge levels with, on average 3 clusters was conducted per block and later simulation was extended for other parameters.

We analyzed the results obtained by the student at the end of the each concept. We diagnosed, if the simulated students with different knowledge levels could answer the questions.

The concepts dealing with difficult details are hidden from students with lower knowledge levels. Whenever the information in the individual student model is updated, the course concept page is upgraded to adapt accordingly. Student is given test based on his Knowledge Level (KL) say for example $(K L=0.3)$, novice student, system will start displaying the questions randomly from subject content that is meant for novice students. If student answers at-least 3 questions out of 5 questions, correctly, system will automatically shift KL to $(\mathrm{KL}+1)$. After this student is updated 
with average level subject contents and related questions. In case student does not answer at least three questions correctly, system automatically shifts $(\mathrm{KL}+1)$ to (KL-1). The same concept is displayed again for student to understand well and test will be conducted again. The test will get terminated either on the student has attempted test and failed even after two attempts (Gwo-Jen Hwang , Fan-Ray Kuo, Peng-Yeng Yin, Kuo-Hsien Chuang 2010). We observe that with Ubiquitous Learning, students learn significantly better and also their knowledge level is systematically improved. . Figure 8 shows example of a temporary student model and its structure. shows how adaptive subject content and test related to that topic is presented to students based on their knowledge level. These characteristics were tested to observe and evaluate the student's performance and improvement, number concepts referred, number of concepts repeated. as seen in (Figure 9) etc. Expert students correctly diagnoses $90 \%$ of concepts, average students correctly diagnose $70 \%$ of concepts and $30 \%$ non correctly and novice students attempt the same concepts and then correctly diagnose $50 \%$ correctly and then upgrade their knowledge.

\begin{tabular}{|lcccccc|}
\multicolumn{8}{c|}{ Student : Novice student KL-0.3 } \\
\hline Student ID & Test ID & Date & KL & Lower KL & Upper KL & $\begin{array}{c}\text { Confidence factor } \\
\text { Improvement }\end{array}$ \\
\hline John & JohnM1 & $4 / 6 / 2015$ & 0.3 & 0.3 & 0.5 & Yes \\
\hline
\end{tabular}

\begin{tabular}{|c|c|c|c|c|c|c|c|c|c|c|}
\hline \multirow[b]{2}{*}{ Student ID } & \multicolumn{10}{|c|}{ Knowledge distribution and Update } \\
\hline & Test ID & $\mathrm{M} 1-\mathrm{C} 1$ & c11 & $\mathrm{c} 12$ & $\mathrm{c} 13$ & c14 & $\mathrm{c} 15$ & $\mathrm{C} 2$ & $\mathrm{c} 21$ & $\mathrm{c} 22$ \\
\hline John & JohnM1 & $\checkmark$ & & & $\checkmark$ & $x$ & $x$ & & $\gamma$ & \\
\hline Weightage & M1-20 & & 30 & 30 & 30 & - & - & & 40 & \\
\hline
\end{tabular}

\begin{tabular}{|l|l|r|r|r|}
\hline Questions Generated \\
\hline Student ID & Q-ID & A-lD & C/W & KL \\
\hline \multirow{7}{*}{ John } & M1,C1 & & & \\
C11 & A11 & C & KL+1 \\
C12 & A12 & W & KL-1 \\
C12 & A12 & C & KL+1 \\
& C13 & A13 & C & KL+1 \\
\hline & M1, C2 & & & KL-1 \\
& C21 & A21 & W & KL-1 \\
& C21 & A21 & W & KL+1 \\
& C21 & A21 & C & \\
& C22 & & & \\
\hline
\end{tabular}

Figure 8 TEMPORARY STUdENT MOdel

FIGURE 9 SUBJECT CONCEPTS DISTRIBUTION BASED ON KNOWLEDGE LEVEL OF STUDENT.

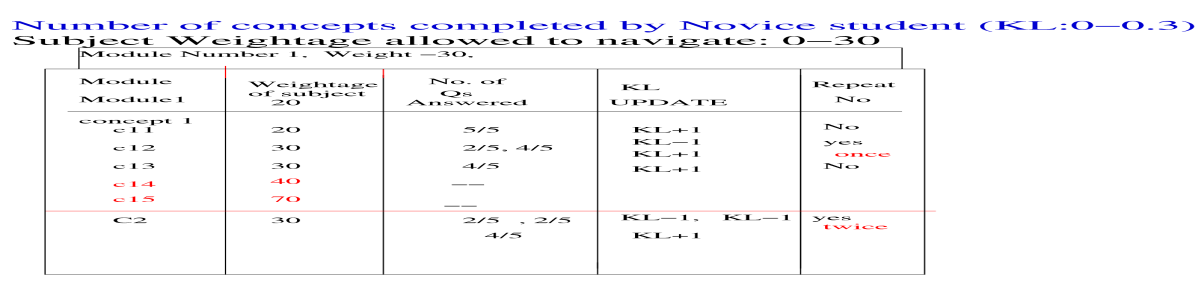


International Journal of Education (IJE), Vol. 3, No. 3, September 2015

\section{CONCLUSIONS}

In this work, we have presented Ubiquitous Learning System based on C-IOB model. Subject contents are well defined and organized in accordance with student's status in real world. The Modules and concepts are interlinked accurately so as to support students with different knowledge levels. We observed that with Ubiquitous Learning system, students learn significantly better and their knowledge level is systematically improved.

\section{REFERENCES}

[1] M.Wis (1993) 'Ubiquitous Computing', Communications Of ACM , Vol.36(1),75-84.

[2] M. Takemoto, et al(2004) 'A Service-Composition and Service-Emergence Framework for UbiquitousComputing Environments' SAINT2004 , 313-318,Jan. 2004..

[3] Sejong Gu, Hongzhou Shi, Jian Ye, Zhenmin ZhuM. Takemoto, et (2007) ' A Service Discovery framework for Ubiquitous Computing', 8th Intl. IEEE 2007.

[4] Pushpa(2011) 'Context aware M-commerce services: C-IOB model approach' ICICS ,13-16 Dec. 2011, Singapore.

[5] Tianyin Xu, Baoliu Y(2007) 'A Gnutella Inspired Ubiquitous Service Discovery Framework for Pervasive Computing Environment' Tokyo 163-0914 JAPAN, 2008 IEEE .

[6] B.Miller and R.Pascoe(2000) 'Salutation Service Discovery in Pervasive Computing' Tokiya2000 1630914, JAPAN, 2008 IEEE.

[7] Okkyung Choi and SangYong Han2005 'Ubiquitous Computing Services Discovery and Execution Using a Novel Intelligent Web Services Algorithm', Sensors 2007 1287-1305, ISSN 1424-8220.

[8] Feng Zhu, Matt W. Mutka and Lionel M. Ni(2005) 'Service Discovery in Pervasive Computing Environments' 1536-1268/05/ 2005 IEEE

[9] Choonhwa Lee, Sumi Helal(2003) 'Context Attributes: An Approach to Enable Context-awareness for Service Discovery' Proceedings of the 2003 Symposium on Applications and the Internet, 2003.

[10] Okkyung Choi and SangYong Ha (2007) "Ubiquitous Computing Services Discovery and Execution Using a Novel

Intelligent Web Services Algorithm' Sensors 2007 , 7, 1287-1305, ISSN 1424-8220.

[11] Kouadri Mostefaoui, S, Tafat-Bouzid, A, Hirsbrunner (2005), 'Using Context Information for Service Discovery and Composition' Proc. Of the 5th Conf. on information integration and web-based applications and services Jakarta, p. 129-138.

[12] Anandi02 'A design of subject model for Web-based education system' IEEE HNMC2004, 313-318, Jan. 2004'

[13] hunc08 'An efficient method for extracting the user profile from dynamic web site using hunc algorithm' Journal of mathematics and technology, 2070-0257, August. 2010.

[14] Eva Millán, *, Luis Descalço , Gladys Castillo , Paula Oliveira , Sandra Diogo , "Using Bayesian networks to improve knowledge assessment," Computers \& Education, Volume 60, Issue 1, January 2013, Pages 436-447

[15] Eva Millána, , , Tomasz Lobodab, Jose Luis Pérez-de-la-Cruza, "Bayesian networks for student model engineering", Computers \& Education, Volume 55, Issue 4, December 2010, Pages 1663-1683.

[16] Gwo-Jen Hwanga, , , Fan-Ray Kuoa, , Peng-Yeng Yinb, , Kuo-Hsien Chuangb, , “A Heuristic Algorithm for planning personalized learning paths for context-aware ubiquitous learning" , Computers \& Education, Volume 54, Issue 2, February 2010, Pages 404-415.

\section{AUTHORS}

\section{First Author.}

Anandi Giridharan, received MSc(Engg) from Indian Institute of Science. She currently working as Senior Scientific Officer in ECE Department, Indian Institute Science, Bangalore. Her Research Interest are in area of Ubiquitous Learning Communication Protocols and Multimedia systems. 


\section{Second Author}

Prof. Venkataram Pallapa received his Ph.D. Degree in Information Sciences from the University of Sheffield, England, in 1986. He is currently the chairman for center for continuing education, and also a Professor in the Department of Electrical Communication Engineering, Indian Institute of Science, Bangalore, India. Dr. Pallapa's research interests are in the areas of Wireless Ubiquitous Networks, Communication Protocols, Computation Intelligence applications in Communication Networks and Multimedia Systems. Dr. Pallapa is the holder of a Distinguished Visitor Diploma from the Orrego University, Trujillo, PERU. He has published over 150 papers in International/national Journals/conferences. Written three books: Mobile and wireless application security, Tata McGraw-Hill, Communication Protocol Engineering, publications Prentice-Hall of India (PHI), New Delhi, 2014 (Coauthor: Sunil Manvi, B Satish Babu) and Multimedia: Concepts \& Communication, Darling Kinderley(India) Pvt. Ltd., licensees of Pearson Education in South Asia, 2006. Written chapters for two different books, and a guest editor to the IISc Journal for a special issue on Multimedia Wireless Networks. He has received best paper awards at GLOBECOM'93 and INM'95 and also CDIL (Communication Devices India Ltd) for a paper published in IETE Journal. He is a Fellow of IEE (England), Fellow of IETE(India) and a Senior member of IEEE Computer Society. 